\title{
Los hipsters bogotanos: visión desde la antropología social en perspectiva publicitaria
}

\section{Hipsters in Bogotá: a view from social anthropology on an advertising perspective}

\author{
José Polanco Contreras (Colombia) \\ Universidad Jorge Tadeo Lozano \\ jose.polanco@utadeo.edu.co
}

\author{
Vladimir Sánchez Riaño (Colombia) \\ Universidad Jorge Tadeo Lozano \\ vladimir.sanchez@utadeo.edu.co
}

\section{Resumen \\ Este artículo recogió algunas \\ investigaciones en torno al fenómeno de la moda en Bogotá, realizadas por el Observatorio de Antropología y Publicidad de la Universidad de Bogotá Jorge Tadeo Lozano. Además de presentar un estado de la cuestión sobre la relación actual de la antropología social y la publicidad, se presenta el caso del grupo social hipsters bogotanos. El método etnográfico de la investigación permite tener hallazgos significativos,}

\begin{abstract}
:
This article resumes the insights gathered from the fashion world in Bogotá, brings to light thanks to the action of the social anthropology observatory, part of the Advertising Program in the Jorge Tadeo Lozano University. In the development of the text, first we expose a framework about the relationship between the social anthropology and the advertising industry also; This paper show the case of the Hipsters, an upcoming subculture in Bogotá. The ethnography method of the
\end{abstract}

Para Citar este artículo / to Cite this article Polanco, J. \& Sánchez, V. (2013). Los hipsters bogotanos: visión desde la antropología social en perspectiva publicitaria. Poliantea, (IX), 17, pp. 89-106. 
como la creciente tendencia a la personalización en el mundo de la moda, la reafirmación de la moda como una forma de expresión y representación social y la eficaz penetración de los nuevos medios, como Internet, para crear tendencias y resistencias a las grandes marcas en los planos tanto locales como globales.

Palabras clave: moda, etnografía, tendencias, publicidad, mercadeo, observatorio, antropología social, prácticas sociales y culturales. research brings the possibility of gain cultural insights like the growing trend based in the social customization of the fashion and the use of the new media such Internet in order to create fashion communities of counter culture.

Keywords: fashion, ethnography, trends, advertising, marketing, observatory, social anthropology, social and cultural practices. 


\section{Los hipsters bogotanos: visión desde la antropología social en perspectiva publicitaria}

\section{Hipsters in Bogotá: a view from social anthropology on an advertising perspective}

José Polanco Contreras (Colombia)

Magíster en Antropología
Vladimir Sánchez Riaño (Colombia)

Magíster en Semiótica

\section{Introducción}

El siguiente artículo tiene como propósito hacer una presentación de algunos hallazgos etnográficos realizados por el Observatorio de tendencias para marcas y comunicación publicitaria, que es parte de la sublínea de investigación de Antropología y Publicidad de la Universidad Jorge Tadeo Lozano. En este artículo se presenta un estado actual de la relación de la antropología social y la publicidad y se muestra el caso de los hipsters bogotanos.

El Observatorio de tendencias ${ }^{1}$ es una idea desarrollada por el Comité de Estudios e Investigación del Programa de Publicidad de la Universidad de Bogotá Jorge Tadeo Lozano, en cabeza del doctor Christian Schrader Valencia. En él se ha contado con la participación de varios estudiantes inscritos como semilleros de investigación, quienes han realizado trabajos de campo para el levantamiento de información, que es la base de lo que aquí

1 El observatorio se puede visitar en www.etnografiarocket.com gracias al diseño y diagramación web realizados por el profesor Carlos Arango, del Programa de Publicidad. 
se presenta. La constitución del observatorio se realizó siguiendo las premisas fundamentales que para ello han planteado la antropología y la comunicación como vehículo para comprender los fenómenos sociales y sus tendencias en el mundo contemporáneo.

En esencia, podríamos decir que un observatorio es una herramienta de seguimiento permanente que busca registrar el acontecer de fenómenos sociales y culturales que previamente han sido definidos de forma clara y concreta en función de unos objetivos de investigación. En el caso de los observatorios que involucran el seguimiento del acontecer publicitario, estos en buena medida se caracterizan por analizar contenidos para "poder en cada caso ensalzar o reprochar públicamente los valores y comportamientos que no se tomen de forma debida" (Pédros, 2005).

En el sentido de hacer un monitoreo crítico de la realidad publicitaria, como lo señalábamos en el párrafo anterior, existen varios tipos de observatorios sobre el tema publicitario, como el de la fundación Ipade, que es una ONG española, que tiene un observatorio enfocado en registrar los mensajes publicitarios con un contenido ecológico. También, en el marco de este propósito crítico podríamos reseñar el Observatorio del Instituto Andaluz de la Mujer, el cual verifica que los mensajes publicitarios no se caractericen por exhibir contenidos sexistas.

Cuando las escuelas que tienen como objeto de estudio los medios se enfocan en describir la actividad de los observatorios, son claras en argumentar que un observatorio debe ir más allá de la actitud crítica para también poder construir un sistema de recolección de informes sobre el acontecer de un fenómeno en los medios y además poder difundir el contenido de estos informes para forjar nuevos puntos de vista sobre este acontecer. De esta forma se busca contribuir a la capacitación de las audiencias en la supervisión de la actividad mediática (Herrera, 2006).

En contraste con la actividad crítica y formadora de opinión de los observatorios que hemos descrito, existen también los que se enfocan en el registro y descripción de tendencias. Este es el caso de los observatorios creados para detectar tendencias que sirvan como insights en el desarrollo de ideas. En esta 
línea de argumentación, se puede citar el caso del observatorio creado por la Cámara de Comercio de Bogotá, en 2008, con el propósito de posicionar internacionalmente los productos de Bogotá y Cundinamarca, a la luz de las tendencias globales y locales sobre el comportamiento del consumidor. Este observatorio es fruto de una alianza estratégica con la empresa Future Concept Lab. Esta alianza ha capacitado a varios coolhunters que han podido sacar a la luz tendencias globales y locales sobre el comportamiento del consumidor.

Future Concept Lab es un laboratorio de investigación que tiene una amplia trayectoria internacional apoyando centros especializados en investigación de tendencias de consumo, con actividades extendidas en Europa, Suramérica y Asia. Es un centro global que tiene sus cuarteles en Milán y su propósito es desarrollar y compartir los nuevos conceptos en el desarrollo de productos, comunicaciones y distribución, con el objetivo de dar a los clientes información sobre el avance de mercados emergentes en relación con los desarrollos claves para el futuro ("Overview Future Concept Lab,” n. d.).
Como lo hace Future Concept Lab, existen otras organizaciones enfocadas en el rastreo de tendencias que publican sus registros de investigación de forma permanente en la web. Se pueden destacar los casos de Trendhunter.com y Trendwatching.com. También se puede usar como referencia el caso de Google Trends, que muestra los términos de búsqueda más populares desde el año 2004, graficando cuáles son las búsquedas y términos globales más buscados y populares en la web.

Los ejemplos que hemos citado, son importantes para enmarcar la actividad del observatorio de antropología y publicidad. De hecho, los casos que se han descrito como ejemplo sirvieron como inspiración para el diseño y montaje del observatorio. Incluso el grupo de trabajo se dio a la tarea de bautizarlo con un nombre que funcionara como una marca atractiva para los estudiantes y en general para la comunidad académica que tuviera contacto con el observatorio. El nombre elegido fue Rocket, inspirado en la filosofía que se resume en su slogan publicitario: "combustible para las ideas". 


\section{Marco teórico}

\section{Comportamiento del consumidor}

El comportamiento del consumidor puede ser descrito como las actitudes, intenciones, decisiones y acciones de los individuos para la toma de decisiones en un campo del mercado. El estudio del comportamiento del consumidor ha contado con la intervención de varias ciencias sociales, como la psicología, la sociología, la antropología, la economía y la historia, todas estas brindan su aporte en la explicación y la predicción de los comportamientos que tienen los consumidores de modo que con estos resultados se puedan implementar acciones de márquetin, comunicación publicitaria y clasificación de las necesidades personales dentro del plano cognitivo, afectivo y en general la dimensión comportamental. Históricamente en la relación del comportamiento del consumidor con la economía, son célebres las visiones críticas de autores como Georg Simmel, Horkehemimer y Adorno (Belch y Belch, 2009; Peter y Olson, 2002; Solomon, 2010).

\section{Coolhunting y antropología social}

En relación con el comportamiento del consumidor y las técnicas que existen para sus estudios podemos mencionar el coolhunting, que adapta en esencia muchos de los fundamentos de la etnografía, con el propósito de registrar tendencias. Víctor Gil en su libro Coolhunting. El arte y la ciencia de descifrar tendencias dice que:

"Hacer coolhunting es saber detectar precozmente la concurrencia de determinados cambios en la esfera social que pueden suponer nuevas necesidades de los consumidores; necesidades, que con toda probabilidad, ni siquiera hayan sido formuladas de forma explícita por estos y que por lo tanto difícilmente van a ser detectadas a través de los mecanismos tradicionales de investigación del consumidor" (Gil, 2009, p. 23).

Esta definición que hace Gil (2009) en su texto, nos introduce a la actividad fundamental de Rocket como observatorio, la cual estuvo basada en el registro de fenómenos culturales que son indicios de tendencias a través de la técnica etnográfica, que es el método por excelencia de la antropología social. 
En los últimos años el auge del análisis antropológico en el mundo de la publicidad y el márquetin se ha convertido en una asesoría en demanda, pues este revela hallazgos que son significativos para los mercados de productos y servicios. Antropólogos y publicistas se concentran en el estudio de la interacción social, en el análisis de creencias y valores que rodean la relación entre las personas, los bienes y la sociedad (Malefyt y Moeran, 2003 , p. 14). En el plano académico podemos señalar que en el año 2010 se publicó por primera vez The international Journal of Business Anthropology.

Sobre la creciente demanda por el análisis antropológico, por ejemplo, Clow y Baack plantean el siguiente caso sobre la relación de la etnografía con el mundo de la publicidad y el márquetin:

"Cuando Whrilpool empleó antropólogos para estudiar lavadoras, se puso de manifiesto que los hombres adultos y los niños también las usaban, y no sólo las esposas y madres. Los investigadores propusieron que los controles de las unidades fueran más sencillos de usar y entender para los hombres y niños, que podían estar menos familiarizados con los ajustes de temperatura y ciclo de lavado más apropiados para cierto tipo de telas" (Clow y Baack, 2010, p. 129).

Del anterior ejemplo se sigue que la etnografía permite adelantar análisis de corte cultural a partir de las investigaciones antropológicas realizadas para el márquetin y la publicidad. En efecto, la etnografía para el mundo del márquetin se ha convertido en una herramienta que permite hacer análisis culturales de productos y marcas, basados en gran medida en la mirada estructuralista de la antropología (Sunderland y Denny, 2007). Mariampolski (2006, p. 5) argumenta que contar con información sobre el comportamiento del consumidor, sus emociones, impresiones y lenguajes en relación con las marcas y categorías de productos, ofrece un punto de partida para la "aventura del marketing".

Para la publicidad y el mercadeo el tema etnográfico ha aparecido en la actualidad como algo novedoso, sobre todo en el ámbito de las agencias de publicidad. Charles Winick, en su artículo de 1961 advertía, adelantándose a los tiempos de hoy, que los investigadores en márquetin hacen una notable investigación antropológica en las culturas modernas en relación con 
el trabajo de los antropólogos, al estudiar subculturas y formas de vida logrando recavar un tipo de información que podría resultar como material de primera línea para la antropología cultural.

Mariampolski (2006) hace una línea de tiempo que resume el papel de la etnografía en la planeación de políticas contra la pobreza y ayuda a arrojar luces en el ámbito de los temas propios de la cultura. La aplicación de la técnica etnográfica en los estudios urbanos contemporáneos, inicia, para Mariampolski, con los trabajos de la escuela de Chicago en los años veinte, constituyendo una nueva etnografía urbana que en los años ochenta comienza a ser aplicada a los estudios del consumidor y a la investigación de márquetin.

\section{Publicidad}

La publicidad, que se define como un "esfuerzo pagado, transmitido por medios masivos de información con el objeto de persuadir" (O`guinn, Allen y Semenick, 1999, p. 6), puede ser vista más allá del simple plano comercial, como lo argumenta Barbara Stern, de la Universidad de Rutgers, quien dice que la publicidad es "una forma de texto literario estructurado, muy distinto de la comunicación espontánea de boca en boca del lenguaje oral" (Arens, 2008, p. 9).

A la razón de que el mensaje publicitario no es una simple construcción que resuelve objetivos de márquetin, como lo expresábamos en el párrafo anterior, podemos agregar que el hecho de la creación publicitaria mantiene una relación fronteriza entre el arte y su forma de comunicación. Joan Gibbons (2008, p. 158) dice que en la publicidad, como en el arte, existe una relación de autoría y de autenticidad que las une, además la publicidad endosa un aura de producción única y preciosa a las piezas que produce.

Si pensamos en la publicidad como una producción en la cual la cultura es una fuerza importante de cohesión en la que se involucran el arte y lo cotidiano, entonces podríamos estar de acuerdo con Malefyt y Moeran (2003, p. 9), quienes argumentan que los profesionales del mundo del márquetin y la publicidad conciben la cultura como una especie de pegamento invisible que une los comportamientos de los consumidores. En esta línea argumentativa adquiere mayor relevancia el pensamiento sociológico que entiende a la publicidad como el eje 
central de la "información posindustrial en la sociedad basada en el conocimiento" (Featherstone, 1991; Beck et al.; Stenberg, 1999; Davis y Scase, 2000, McFall, 2004).

Bourdieu (1984) pensaba que la publicidad, como el diseño gráfico, el diseño de empaques, las promociones de ventas y en general todos los actores involucrados en la comunicación de márquetin, desempeñaban un papel de "intermediarios culturales" en el ámbito pequeño-burgués, como los creadores de necesidades y operadores de un aparato simbólico en torno a los bienes y servicios producidos.

Lo interesante de la publicidad como actor social e intermediario cultural, es que es objeto de críticas $y$ se convierte en una figura de primera línea cuando se trata de hablar de comunicación, tendencias y cultura popular. Arens argumenta que "los críticos han denigrado a la publicidad por una amplia gama de pecados, algunos reales y otros imaginados". Al respecto cita las palabras de John O'Toole, quien fue presidente de Foote, Cone \& Belding y presidente de la Asociación Estadounidense de Agencias de Publicidad, quien decía que "[...] Muchos críticos atacan la publicidad porque no es otra cosa. No es periodismo, ni educación, ni entretenimiento, aunque con frecuencia realiza las tareas de los tres" (2008, p. 57).

No admite discusión el papel de la publicidad en la cultura y de la cultura en la publicidad. Al respecto, Mattelart (1991) argumentaba que la cultura no se puede considerar como un terreno descontaminado, pues está de alguna forma colonizado y mercantilizado por las prácticas de la publicidad y el mercadeo. Para Lash y Urry (1994) existe un ambiente cultural que es producido por las nuevas combinaciones entre los sistemas económicos y los entornos culturales, que es construido por el trabajo de producción simbólica de la publicidad.

Para hablar de la participación de la cultura en la publicidad y la necesidad de estudiarla a través del método etnográfico y los observatorios de tendencias y publicidad, podemos ver cómo hoy en día muchos de los mensajes publicitarios que se producen ya no provienen de los departamentos creativos de las agencias de publicidad, como era la vieja usanza, sino que son los consumidores los que generan el contenido de los mensajes. Clow y Baack (2010, p. 256) presentan varios casos 
sobre la participación de la cultura en publicidad:

"En el Super Tazón de 2007, Doritos presentó un comercial que era el resultado de un concurso de creación de anuncios entre consumidores, Converse, Firefox y Diet Coke también han usado materiales generados por los consumidores [...] La idea principal es que los espectadores del anuncio tienden a aceptar mejor un anuncio generado por un consumidor porque lo consideran más genuino que un comercial producido por la empresa".

Con lo anterior se evidencia la estrecha y recíproca relación entre la cultura y la publicidad, la cual ha catapultado tanto a la antropología social como a la etnografía enfocada a los negocios, como un campo relevante de trabajo en la planeación publicitaria de hoy. Este papel protagónico es justamente lo que justifica el trabajo de los observatorios culturales como entes de registro de tendencias para la obtención de insights publicitarios, gracias a que trabajan como verdaderos sensores culturales que posibilitan que la actividad de la publicidad pueda ser entendida como "espejo de la sociedad" (Sivulka, 2004).

Sunderland y Denny (2007) argumentan que hoy "el uso de la etnografía se ha convertido en una clase de Santo Grial en la búsqueda de formas para la venta de las marcas" (p. 26), lo que resulta ideal para ellas, pues como lo dice Geertz (1977), la antropología nos ha enseñado a reconocer la capacidad humana de girar, invertir, inventar y variar las cosas con los significados que le damos al mundo, y en resumidas cuentas qué es una marca sino "una historia que nunca se acaba de contar" (Mariotti, 2001) con miles de posibilidades de cambios, giros e inversiones simbólicas.

Malefyt y Morais (2012, p. 12), dicen que hoy existe una presión por conducir negocios en un entorno global que ha creado "un permanente estado de emergencia" (Thrift, 2000 , p. 674) que requiere de disciplinas que puedan estar al tanto de los cambios que se producen en la cultura; existe una imperiosa necesidad de innovación y creatividad para la agencias de publicidad que buscan mejores formas para llevar los mensajes publicitarios a los consumidores. Para ellos esta es la razón para que exista la importante presencia de la antropología cultural y de las técnicas etnográficas en la vida de la empresa moderna. Sobre el particular Malefyt y Morais afirman: 
"Para los antropólogos del mundo de los negocios, leer y escribir sobre la cultura es uno de los componentes de un multifacético proceso interactivo dentro de las redes de las relaciones humanas que comprenden el mundo de la publicidad. Los antropólogos en el ámbito de lo comercial y de los negocios responden por la vidas y las reacciones en torno a las marcas y colaboran con colegas para trasponer los hallazgos en estrategias, planes de mercadeo y eventualmente en el trabajo creativo" (2012, p. 10).

La importancia, aplicación y desarrollo de la antropología social y en particular de la etnografía en el ámbito de la publicidad, que se ha puesto de manifiesto, se evidencia en el presente artículo, en el que se resumen los hallazgos del trabajo de campo en torno al fenómeno de los hipsters, realizados por los grupos de trabajo pertenecientes al semillero de investigación de Rocket.

\section{Metodología}

Para la tarea de la caza de tendencias y la comprensión de los fenómenos culturales se utiliza la técnica etnográfica, que según Guber, "es una concepción y práctica de conocimiento que busca comprender los fenómenos sociales desde las perspectivas de sus miembros entendidos como actores, agentes o sujetos sociales" (Guber, 2007 [2001], pp. 12-13). Por ejemplo, Nabisco descubrió gracias a dicha técnica, que hoy en día las mujeres de mediana edad tienen un "sentido elevado de merecimiento propio". Ya no se trata de verse bien en un traje de baño, sino de la experiencia de aquello que se ajusta con lo que me merezco, con ello las dimensiones emocionales y aspiracionales de los individuos son develadas por los estudios etnográficos. La agencia Foote, Cone \& Belding supo capitalizar este hallazgo con el mensaje: "Comer bocadillos no es sobre llenarse, sino sobre satisfacerse" (Arens, 2008, p. 154).

Teniendo en cuenta dicha metodología el observatorio hace estudios de corte cualitativo con análisis de inductivo de datos, desde la perspectiva metodológica $y$ analítica propia de la visión de la antropología social, la cual desde una tradición constructivista, le permite a los investigadores darle sentido a los significados que otros tienen del mundo (Creswell, 2007).

Ahora bien, la elección de las poblaciones de estudio se ha hecho apelando a la metodología de muestreo por conveniencia (Ritchie y Lewis, 2003) y se ha utilizado la técnica de Snowball Referral (Bailey, 
1994) para ponerse en contacto con los informantes que le dan cuerpo al trabajo de campo. Se incluyeron en el estudio todas las personas habitantes de Bogotá que hacen parte de fenómenos de tendencias culturales y se excluyeron todas aquellas que generaban un conflicto de intereses. Algunos nombres de los entrevistados han sido cambiados para salvaguardar la privacidad de los participantes en la investigación $\mathrm{y}$ todos los involucrados fueron informados por los investigadores de la naturaleza académica del trabajo. Los instrumentos de recolección de la información han sido: entrevistas a profundidad, diarios de campo con registro en audio y video.

El motivo por el que se ha tomado la moda como eje central para la composición de este artículo, es la forma compleja como esta es influida por valores estéticos y morales en lo concerniente a la conducta individual y social como vehículo y motor de las transformaciones culturales, evidenciables en los subgrupos urbanos que a su vez se convierten en objetos de estudio para la identificación de tendencias aprovechables en el mundo de la publicidad. Hillmann (2005, p. 587) dice que "la difusión universal del fenómeno de la moda induce cada vez más a investigar las fuerzas motrices humanas fundamentales, como la publicidad, que intervienen en ella”.

\section{Hallazgos: el caso de los hipsters bogotanos}

El término hipster se ha utilizado para designar a esta "tribu urbana” (Maffesoli, 1996) y empezó a ser usado en la década de los noventa en los Estados Unidos para referirse a jóvenes de clase mediaalta que cumplían con ciertas características. Por ejemplo, su gusto por la música independiente (indierock), la fotografía, el arte, la literatura, la comida orgánica y el cine independiente.

La investigación encontró que los hipsters al ser una subcultura alejada de lo convencional, no siguen tendencias específicas de moda, sino que hacen una combinación muy propia y personalizada de estas para crear un estilo totalmente nuevo. Por ejemplo, argumentan los investigadores como notas de su diario de campo:

"La pinta de un hipster puede incluir una camisa Burberry de 700 dólares, unos jeans Levi’s de 50 dólares y unos tennis Converse rotos. También es interesante cómo la ropa es un elemento que les permite exteriorizar su lado intelectual al 
combinar un look nerd con un toque moderno: unas gafas de pasta o una camisa de botones pueden ir perfectamente con unos jeans rotos y unas botas militares".

Los resultados de la investigación muestran que para esta subcultura lo intelectual y el gusto por la moda no son dos ítems excluyentes y es precisamente este aspecto lo que los hace tan atractivos como grupo de investigación, su visión ecléctica de la moda y el estilo, los proyecta como creadores de tendencias.

Con la intención de crear una historia de vida que permitiera ampliar los resultados de la parte exploratoria de la investigación, el grupo acompañó durante un día a David Echeverri, de 22 años, estudiante de arquitectura en la Universidad de los Andes, para registrar en video los acontecimientos más llamativos en la vida de un hipster bogotano.

Los siguientes son apartes del diario de campo que surgió del análisis del video registrado por los investigadores:

El día comenzó cuando a la 1 p. m. llegamos a su casa, nos ofreció pasta integral con Pesto y así comenzamos a conversar un poco. Nos habló de que en la noche debía asistir a una galería de arte al lanzamiento del fanzine ${ }^{2}$ de uno de sus amigos. Durante el día sus actividades incluyeron navegar en la web, salir al parque a leer, escoger la ropa para el evento, montar en su bicicleta vintage y comer cupcakes y espresso en una panadería cerca a su casa. Le hicimos varias preguntas acerca de sus gustos por marcas, las páginas que visitaba y los lugares que frecuentaba y concluimos que para él uno de los elementos más influyentes a la hora de vestirse o comprar ropa era Internet, ya que visitaba blogs para ver qué estaba de moda en las calles de Nueva York y otras ciudades, con el propósito de decidir qué artículos podía incluir en su clóset.

El grupo de investigación también visitó la zona de la 54 con 7. ${ }^{\mathrm{a}}$, en el barrio Chapinero. Este sector se ha convertido en un punto de referencia de la moda bogotana para personas como los hipsters. Estos lugares son tiendas independientes, creadas por jóvenes diseñadores con una visión contemporánea de la moda que se ve reflejada en sus diseños.

Se realizaron entrevistas semiestructuradas a los vendedores de las

2 Los fanzines son publicaciones no profesionales producidas por seguidores de un fenómeno cultural particular, como puede ser un género literario, musical, el cómic, etc. 
tiendas Resistencia, Juan, John Bandera y Perfes; a partir de la información recolectada el grupo encontró que estos espacios comerciales manejan como grupo objetivo un mercado variado en el que se encuentra la gran mayoría de tribus urbanas, incluido el grupo protagonista de esta investigación: los hipsters.

La oferta comercial que atrae a los hipsters está basada en la exclusividad de las prendas que allí se pueden comprar. En esta zona de diseño se encuentran prendas y accesorios clásicos con toques modernos que permiten dar ese significado de "único" a las personas que los usan.

Solo hasta hace unos años este tipo de tiendas independientes empezaron a tener reconocimiento en la moda bogotana y se considera que son un punto importante a la hora de definir el estilo de los hipsters, ya que son establecimientos donde se encuentran varias marcas, accesorios, cámaras fotográficas, zapatos importados y camisetas diseñadas por jóvenes talentos.

A través del diario de campo se logró identificar la importancia, los almacenes y la cultura que allí se moviliza. A continuación se presenta un fragmento del diario de campo que da cuenta de ello:
Viviana Lozano es una de las propietarias de la tienda de ropa Juan, ubicada en la calle 54 con 7. a. Esta tienda es uno de los espacios que más acogida ha tenido dentro de la moda local, ya que sus diseños evocan un estilo cosmopolita y urbano. Al preguntarle a Viviana sobre los hipsters, a quienes considera parte de su grupo objetivo, nos dijo que una de sus principales referencias a la hora de vestirse eran los blogs de moda y las personas que dentro de ellos son las más "rankeadas". También nos pareció interesante el hecho de que Juan hace su publicidad utilizando jóvenes comunes, estudiantes de universidad y no grandes modelos de agencia. Este enfoque publicitario es un gran acierto ya que es más cercano al público bogotano y no tan impersonal como los anuncios de Zara, en los que vemos modelos suecos vistiendo abrigos de piel. Para Viviana Lozano este punto fue fundamental en la creación de su campaña, ya que en ella se quiso mostrar gente real y jóvenes de la vida cotidiana promocionando tendencias y estilos para crear un vínculo más fuerte con la audiencia, haciéndola también parte del proyecto y dándole la libertad de ser parte de él. 
En las conclusiones del trabajo de campo, el grupo investigador dice que el hipster bogotano se acerca bastante a lo que es el modelo de hipster estadounidense y especialmente al neoyorquino, ya que fue una subcultura que se difundió gracias a Internet, a través de revistas virtuales y blogs. El hipster criollo adoptó principalmente la forma de vestir y de forma secundaria las corrientes musicales y artísticas. Para ellos es claro que la escena local está influenciada por las corrientes internacionales y la herramienta más eficaz para enterarse de las tendencias actuales es la web.

El grupo destaca la participación de Andrea Arbeláez, una de las creadoras de la feria de moda y diseño "Las Puertas de Cielo". Su testimonio es útil como ejemplo del acontecer del movimiento hipster en Bogotá. El proyecto de Andrea, básicamente, se trata de un evento bimensual en el que jóvenes diseñadores y marcas independientes de ropa y accesorios se reúnen en el parque El Chicó y forman pequeñas tiendas al estilo mercado de las pulgas para vender y difundir sus marcas. Esta feria, creada hace aproximadamente un año en la ciudad de Bogotá, es un espacio lleno de innovación y sobre todo de tendencias que se pueden considerar como uno de los principales eventos a los que asiste el hipster bogotano.

\section{Conclusiones}

En suma, cinco ideas y un pronóstico que salen del análisis de esta investigación:

Primero, la moda hoy en día ha pasado de ser un factor de diferenciación social a una forma de representación social que se desliga incluso de la operación de las grandes marcas. Segundo, la moda es común a todos los grupos sociales y puede que sea más poderosa como efecto cultural en los grupos que se vinculan con ella, que la misma imagen que se desprende de los anuncios publicitarios. E incluso podría inferirse que es más relevante que los mismos diseños que se producen en el mundo de la moda. Tercero, las subculturas que se crean en torno a la moda han pasado a ser una manifestación de estados de ánimo de los individuos y de formas de ser ante el mundo. Una prenda hoy en día es una declaración de independencia en la sociedad. Cuarto, la web y la comunicación de voz a voz sirven a las marcas globales, pero también le imprimen dinámicas propias a los 
mercados locales, que hoy por hoy mezclan patrones, diseños y marcas gracias a la gran cantidad de información cambiante que circula por la web. Quinto, una posibilidad interesante para la diferenciación en mercados cada vez más competidos por actores globales con gran poder económico es la customización ${ }^{3}$ de los productos de moda y la siempre efectiva atención personalizada.

\section{Pronóstico}

A futuro la actividad publicitaria en los mercados de moda estará más a la orden de la creación de espacios para compartir estilos de vida, que para perpetuar el discurso hegemónico de los mensajes publicitarios de siempre. Un ejemplo de esto es que grandes casas de moda, como la propietaria de la marca Zara, tiene diferentes formatos de tiendas que se ajustan a los subgrupos y tribus que se crean en torno a la moda. La idea es que cada grupo tenga su propia casa, su propio espacio comercial y no como pasaba antes, que se limitaba a tener un espacio publicitario donde entraban todos

3 Término del márquetin para designar la personalización de productos y servicios. los públicos, como es el caso de los medios tradicionales.

\section{Referencias}

Arens, W. F. (2008). Publicidad. México: McGraw-Hill.

Atkinson, R. (1998). The life story interview. Thousand Oaks, CA: Sage Publications.

Bailey, K. D. (1994). Methods in social research. Nueva York: Free Press.

Beck, U.; Giddens, A. y Lash, S. (1994). Reflexive modernization: politics, tradition and aesthetics in the modern social order. Stanford, CA: Stanford University Press.

Belch, G. E. y Belch, M. A. (2009). Advertising and promotion: an integrated marketing communications perspective. Boston: McGraw-Hill Irwin.

Bourdieu, P. (1984). Distinction: a social critique of the judgement of taste. Cambridge, MA: Harvard University Press.

Clow, K. E. y Baack, D. E. (2010). Publicidad y comunicación integral de marca (4. ${ }^{\mathrm{a}}$ ed.). Nueva Jersey: Pearson Hall. 
Creswell, J. W. (2007). Qualitative inquiry and research design: choosing among five traditions. Thousand Oaks: Sage Publications.

Davis, H. y Scase, R. (2000). Managing creativity: the dynamics of work and organization. Buckingham: Open University Press.

Featherstone, M. (1991). Consumer culture and postmodernism. Los Ángeles: Sage Publications.

Geertz, C. (1977). The interpretation of cultures. Nueva York: Basic Books.

Gibbons, J. (2008). Contemporary art and memory: images of recollection and remembrance. Londres: I. B. Tauris.

Gil, V. (2009). Coolhunting. Madrid: Empresa Activa.

Guber, R. (2007). La etnografía: método, campo y reflexividad. Bogotá: Norma.

Herrera, S. (2006). El porqué de los observatorios de medios latinoamericanos. Revista Latina de Comunicación Social, 61. Recuperado de http://www.ull.es/publicaciones/ latina/200621HerreraS.htm
Hillmann, K. (2005). Diccionario enciclopédico de sociología. Barcelona: Herder.

Lash, S. y Urry, J. (1994). Economies of signs and space. Londres: Sage.

Maffesoli, M. (1996). The time of the tribes: the decline of individualism in mass society. Londres: Sage.

Malefyt, T. D. y Moeran, B. (2003). Advertising cultures. Oxford: BERG. Malefyt, T. D. y Morais, R. J. (2012). Advertising and anthropology: ethnographic practice and cultural perspectives. Londres: Berg.

Mariampolski, H. (2006). Ethnography for marketers: a guide to consumer immersion. Thousand Oaks, CA: SAGE Publications.

Mariotti, J. L. (2001). Smart: lo fundamental y más efectivo acerca de las marcas y el branding. Bogotá: McGraw-Hill.

Mattelart, A. (1991). Advertising international: the privatisation of $\mathrm{pu}$ blic space. Londres: Routledge.

McFall, L. (2004). Advertising: a cultural economy. SAGE.

O’Guinn, T. C.; Allen, C. T. y Semenik, R. J. (1999). Publicidad y 
comunicación integral de marca. American advertising. Belmont, CA: México: Thomson. Wadsworth Pub.

Overview Future Concept Lab (s. f.). Solomon, M. R. (2010). Consumer Future concept Lab. Recuperado de behavior: buying, having, and being. http://www.futureconceptlab.com/ Nueva Jersey: Pearson Education.

Pedrós, G. (2005). Observato- Sternberg, E. (1999). The economy rio de la publicidad de la movili- of icons: how business manufactures dad sostenible y la televisión. Re- meaning. Westport, CT: Praeger. vista Comunicar, 25. Recuperado de http://redalyc.uaemex.mx/ pdf/158/15825088.pdf

Peter, J. P. y Olson, J. C. (2002). Consumer behavior and marketing strategy. Boston: McGraw-Hill.

Ritchie, J. y Lewis, J. (2003). Qualitative research practice: a guide for social science students and researchers. Londres: Sage Publications.

Sivulka, J. (1998). Soap, sex, and Sunderland, P. L. y Denny, R. M. (2007). Doing anthropology in consumer research. Walnut Creek, CA: Left Coast Press.

Thrift, N. (2000). Performing cultures in the new economy. Annals of the Association of American Geographers, 90(4), 674-692.

Winick, C. (1961). Anthropology's contributions to marketing. Journal of Marketing, 25(5), 53-60.

cigarettes: a cultural history of 